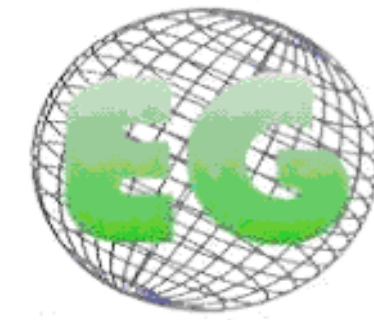

ISSN 1695-6141

$N^{\circ} 23$
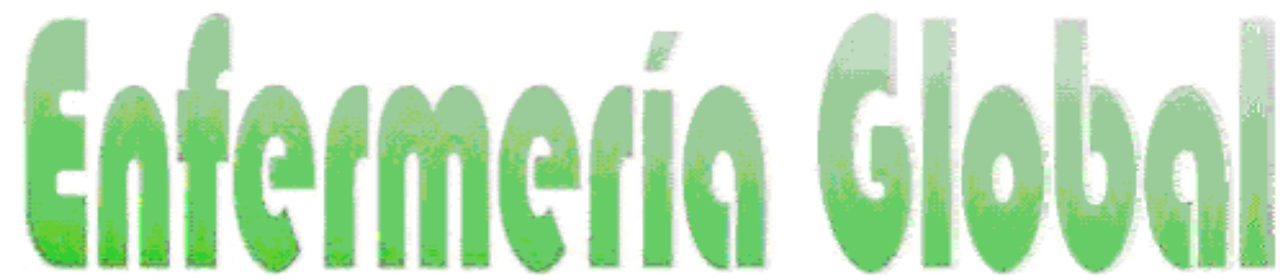

Revista electrónica trimestral de Enfermería

www.um.es/egloball

REVISIONES

\title{
Actuación del enfermero en el control de endemias
}

Atuação do enfermeiro no controle de endemias

\section{${ }^{*}$ De Souza Braga, AL., ${ }^{* *}$ Antunes Cortez, E., ${ }^{* * *}$ Roza Carneiro, F., ${ }^{* * * *}$ Martins Jr., WdS.}

\begin{abstract}
*Mestre - Profesor Asistente de la Escuela de Enfermería Aurora de Afonso Costa - EEAAC. Universidad Federal Fluminense - UFF. ${ }^{* *}$ Doctora- Profesora Adjunta de la Escuela de Enfermería Aurora de Afonso Costa - EEAAC. Universidad Federal Fluminense - UFF. ${ }^{* * *}$ Enfermera de la Casa de Saúde e Maternidade Santa Martha. Posgraduada en Enfermería del Trabajo. ${ }^{* * * *}$ Enfermero del Hospital Quinta Door. Posgraduado en Enfermería del Trabajo. Brasil.
\end{abstract}

Palabras clave: Endemia; Enfermero: Control.

Palavras-chave: Endemia; Enfermeiro; Controle-

Keywords: Endemic disease; Nurse; Control..

\section{RESUMEN}

Endemia es la enfermedad habitualmente presente entre los miembros de un determinado grupo, en una determinada área, en una población definida. El problema del estudio fue reconocer cómo el enfermero actúa en el control de las endemias. El objetivo es identificar la actuación del enfermero en el control de endemias y discutir la formación profesional del enfermero para actuar en dicho control. Estudio descriptivo, exploratorio, cualitativo y bibliográfico, realizado en la base de datos de BVS (BDENF e, LILACS) y en la biblioteca de COC (FIOCRUZ). Tras la colecta de datos se realizó la pre-lectura, la lectura selectiva, la interpretativa y el análisis textual. Emergieron las categorías: el papel del enfermero en el control de las endemias, y la formación profesional del enfermero y el control de las endemias. Los resultados: el papel del enfermero es realizar acciones educativas y administrativas, además de elaborar programas. En cuanto a su formación esta debe enfatizar la importancia de la epidemiología para ser aplicada a la prática profesional. Se concluye que el enfermero orientado a esa función deberá proveer de técnicas de prevención y promoción de la salud procurando una mejor calidad de vida para la comunidad y que en la formación de este deberá desarrollar habilidades técnicas y conocimiento para controlar las endemias. 


\section{RESUMO}

Endemia refere-se à doença habitualmente presente entre os membros de um determinado grupo, em uma determinada área, presente em uma população definida. O problema de estudo foi reconhecer como o enfermeiro atua no controle das endemias? O objetivo é identificar a atuação do enfermeiro no controle de endemias e discutir a formação profissional do enfermeiro para atuar no controle das endemias. Estudo descritivo, exploratório, qualitativo e bibliográfico, realizado na base de dados da BVS (BDENF e, LILACS) e na biblioteca da COC (FIOCRUZ). Após a coleta dos dados realizou-se a pré-leitura, a leitura seletiva, a interpretativa e a análise textual. Emergiram as categorias: o papel do enfermeiro no controle das endemias, e a formação profissional do enfermeiro e o controle das endemias. Os resultados: o papel do enfermeiro é realizar ações educativas e administrativas, além de elaborar programas. Quanto sua formação esta deve enfatizar a importância da epidemiologia para ser aplicada à prática profissional. Conclui-se que o enfermeiro voltado para essa função deverá prover de técnicas de prevenção e promoção da saúde, visando uma melhor qualidade de vida para a comunidade e que na formação deste deverá desenvolver habilidades técnicas e conhecimento para controlar as endemias.

\section{ABSTRACT}

Endemic refers to the disease usually present among the members of a particular group in a given area, that is, present in a specific population. The study problem is: how do nurses operate in the control of endemic diseases? The goal is to identify the role of a nurse in the control of endemic diseases and discuss the training of nurses to operate in the control of endemic diseases. A descriptive, exploratory, qualitative and bibliographic study using the database of the BVS (BDENF, and LILACS) and the COC library (Fiocruz). After collecting data, pre-reading, selective reading, interpretative reading and textual analysis were carried out. The following categories emerged: The role of nurses in the control of endemic diseases, and the professional training of nurses and control of endemic diseases. Results: the role of nurses is to carry out educational and administrative activities, besides elaborating educational programs. When it comes to their training, the epidemic importance to be applied to practical experience should be emphasized. It is concluded that the nurse in charge of this area should provide techniques of prevention and promotion of health, aimed at a better quality of life for the community and that in professional training, the nurse should develop technical abilities and knowledge to control endemics.

\section{INTRODUCCIÓN}

El presente estudio tiene como objeto la actuación del enfermero en el trabajo educativo y preventivo de las enfermedades endémicas.

De acuerdo con Medronho ${ }^{1}$, endemia es la presencia usual de una enfermedad, dentro de los límites esperados, en una determinada área geográfica, por un período de tiempo ilimitado. Este fenómeno ocurre cuando hay una constante renovación de susceptibles en la comunidad, tales como exposición múltiple y repetida de esta a un determinado agente, aislamiento relativo sin desplazamiento importante de la población en una zona territorial.

El análisis de la distribución de las enfermedades y de sus determinantes en las poblaciones en el espacio y en el tiempo es un aspecto fundamental de la epidemiología que, de acuerdo con Rouquayrol ${ }^{2}$, es la ciencia que estudia la distribución de las enfermedades y sus causas en poblaciones humanas.

La justificativa de esta investigación reside en la necesidad de hacer reflexionar al enfermero sobre el tema propuesto para un trabajo educativo y continuado de prevención de 
endemias en el sentido de adoptar medidas eficaces de control. Con ello, surge el problema de estudio: ¿Cómo actúa el enfermero en el control de endemias?

La mayoría de los estudios epidemiológicos consiste en los exámenes cuidadosos de tres cuestiones primordiales: ¿Quién enfermó? ¿Dónde ocurrió la enfermedad? ¿Cuándo ocurrió? Con la primera, se analiza la distribución de la enfermedad según sexo, edad, ocupación, hábitos alimentarios, culturales, etc.; con la segunda, la ocurrencia de algún estándar espacial de la enfermedad, y con la última, el periodo y la velocidad de ocurrencia de la misma. Como resultado de la tercera, podemos clasificarlas en cuanto a la variación en la incidencia de una enfermedad, cuyos ciclos coinciden con las estaciones del año, es denominada variación estacional. Esta variación tiene lugar en un período de un año. Las enfermedades infecciosas agudas constituyen un ejemplo claro de esas variaciones.

La variación estacional depende de un conjunto de factores, tales como: radiaciones solares, temperatura, humedad del aire, precipitaciones concentración de polución en el aire, etc. Además de las condiciones climáticas, una mayor aglomeración climática puede favorecer la aparición de diversas enfermedades respiratorias (por ejemplo, gripe), o un mayor consumo de agua en verano (con el consecuente aumento de los vertidos de aguas residuales) puede favorecer la transmisión fecal-oral (por ejemplo, poliomelitis, diarreas infecciosas, etc. ${ }^{1}$ ).

Así, se propuso como objetivo identificar la actuación del enfermero en el control de endemias y discutir la formación profesional de este para actuar en dicho control.

La salud pública brasileña, antes de la República, estaba repleta de medidas de intervención ambiental, casi siempre en las ciudades, siendo la mayoría de la población rural. Destaca que la situación de los cementerios y hospitales, el drenaje de los terrenos y la influencia de los vientos y hasta de personas nocivas, como: mendigos, enfermos mentales o leprosos constituyó siempre un punto central de preocupación ${ }^{3-5}$.

En el inicio del siglo XX, hubo una sucesión de estudios sobre la etiología, además de la ocurrencia de otros aspectos de diferentes enfermedades endémicas brasileñas, como los estudios de Gaspar Vianna sobre la leishmaniasis cutánea, de Lutz sobre el blasto micose sudamericano y el descubrimiento de la enfermedad de Chagas, en 1909. Este gran movimiento científico concentrado en Rio de Janeiro y São Paulo se hizo notar sobre el control de las enfermedades. La fiebre amarilla, que venía causando epidemias sucesivas en Rio de Janeiro desde 1849 determinó la más emblemática de las acciones de control de endemias en la historia del país ${ }^{4-6}$.

Las acciones de control de endemias fueron perdiendo su importancia en la lógica oficial, aunque fuesen mantenidas, mas no con la prioridad dada en el inicio de la década de 1950, tanto que el Aedes egypti, erradicado en 1955, regresó al país en diversas ocasiones, pero siempre eliminado, hasta que, en 1973, se constata la reinfestación del país, no siendo ya alcanzada su erradicación ${ }^{3}$.

La investigación contribuirá sirviendo como referencia a otros estudiantes, profesionales e investigadores, que adopten la línea de investigación salud y sociedad, teninedo como área predominante enfermería en salud colectiva. Servirá como ayuda para mejorar la construcción del conocimiento de los futuros profesionales de enfermería y para facilitar futuras consultas. 


\section{METOdOLOGÍA}

De acuerdo con los objetivos, este estudio se clasifica en descriptivo y exploratorio. El estudio descriptivo tiene como principal objetivo la descripción de las características de determinada población o fenómeno, y el exploratorio abarca el levantamiento bibliográfico o entrevista con personas que tuvieran experiencias prácticas con el problema estudiado ${ }^{7}$. El enfoque utilizado fue el cualitativo. Este describe la complejidad de una determinada hipótesis o problema, procurando descubrir y clasificar la relación entre variables ${ }^{8}$.

El estudio fue realizado a través de revisión literária en la biblioteca virtual de salud (BVS), en las bases de datos de Lilacs, Bdenf y en la Biblioteca da Casa de Osvaldo Cruz (COC) en FIOCRUZ. Destaca que la revisión tiene como propósito orientar sobre qué es y qué no es conocido en un área de investigación, para confirmar cuál es la mejor investigación que puede deparar mayor contribución al conocimiento ${ }^{9}$.

Utilizamos los descriptores enfermero, endemias y control para la busca. Para organizar el material colectado, optamos por iniciar, selecionando los artigos de acuerdo con los descriptores individualmente, conforme Cuadro 1:

\begin{tabular}{|l|l|l|l|l|}
\hline Descriptores & BDENF & LILACS & COC & Total \\
\hline Endemia & 4 & 433 & 15 & 452 \\
\hline Enfermero & 2112 & 2116 & 2 & 4230 \\
\hline Control & 966 & 42489 & 300 & 43755 \\
\hline
\end{tabular}

Cuadro 1: Distribución cuantitativa de las bibliografías encontradas en las bases de datos

Tras la colecta de datos, percibimos que sería necesario un refinamiento, y optamos por realizar la busca con los descriptores en doble, conforme Cuadro 2.

\begin{tabular}{|l|l|l|l|l|}
\hline Descriptores & BDENF & LILACS & COC & Total \\
\hline Control + Endemias & 2 & 291 & - & 293 \\
\hline Enfermero + Endemias & 2 & 3 & - & 5 \\
\hline Enfermero + Control & 115 & 170 & - & 285 \\
\hline
\end{tabular}

Cuadro 2: Distribución cuantitativa de las bibliografías encontradas en las bases de datos

Partiendo de estos resultados, realizamos una pre-lectura. De acuerdo con Cervo y Bervian ${ }^{10}$ en esta fase inicial de la lectura, el estudiante debe certificarse de la existencia de las informaciones que procura, además de obtener uma visión global de las mismas. Son dos, pues, las finalidades de esta lectura: la primera es permitir seleccionar los documentos bibliográficos que contienen datos o informaciones susceptibles de ser aprovechados en la fundamentación de su trabajo; la segunda es dar una visión global del asunto focalizado, visión indeterminada, mas indispensable para poder avanzar el conocimiento. Se realizó también la lectura selectiva, seleccionando las informaciones que interesan en la elaboración 
del trabajo en perspectiva ${ }^{11}$. De esta manera, la bibliografia potencial fue de cinco trabajos científicos conforme el cuadro ${ }^{3}$.

\begin{tabular}{|l|l|l|l|l|}
\hline Descriptores & BDENF & LILACS & COC & Total \\
\hline Control + endemias & - & 2 & - & 2 \\
\hline Enfermero + endemia & - & - & - & - \\
\hline Enfermero + control & 2 & 1 & - & 3 \\
\hline
\end{tabular}

Cuadro 3 : Distribución cuantitativa de las bibliografías seleccionadas- bibliografia potencial

Finalmente, tras la selección de la bibliografia potencial fue realizada lectura interpretativa, relacionando lo que el autor afirma con el problema para el que se propone una solucción, y, finalizamos con análisis textual que tiene por objetivo una visión global, señalando el estilo, vocabulario, hechos, doctrinas, épocas, autor, o sea, un levantamiento de los elementos importantes del texto ${ }^{12}$.

Tras el análisis, aparecieron dos categorías: el papel del enfermero em el control de las endemias, y la formación profesional del enfermero y el control de las endemias. Resalta que algunos autores fueron categorizados en las dos categorías.

\section{Descripción y discusión de los datos}

\section{1 - El papel del enfermero en el control de endemias}

En esta categoria se incluyen tres artículos y un manual que destacan el papel del enfermero en el controle de endemias, o sea, técnicas utilizadas, acciones educativas, elaboración de programas y gerenciamiento.

\begin{tabular}{|l|c|c|l|}
\hline \multicolumn{1}{|c|}{ Autores (s) } & Año & Base de datos/Revista & \multicolumn{1}{|c|}{ Título } \\
\hline Gomes $^{13}$ & 1994 & $\begin{array}{c}\text { Lilacs/ Rev. Lat. } \\
\text { Americana. }\end{array}$ & A Epidemiologia para o Enfermeiro \\
\hline $\begin{array}{l}\text { Perdesolli, } \\
\text { Antonialli, e } \\
\text { Vila }{ }^{14}\end{array}$ & 1998 & $\begin{array}{c}\text { BDENF/ Rev. Lat. } \\
\text { Americana. }\end{array}$ & $\begin{array}{l}\text { O enfermeiro na vigilância } \\
\text { Epidemiológica no Município de } \\
\text { Ribeirão Preto }\end{array}$ \\
\hline $\begin{array}{l}\text { Ministério da } \\
\text { saúde }\end{array}$ & 2002 & $\begin{array}{c}\text { Ministério da Saúde/ } \\
\text { Fundação Nacional de } \\
\text { Saúde }\end{array}$ & $\begin{array}{l}\text { Ações de Controle de Endemias/ } \\
\text { Manual de Agente Comunitário de } \\
\text { Saúde e Agente de Controle de } \\
\text { Endemias }\end{array}$ \\
\hline $\begin{array}{l}\text { Nichiata, } \\
\text { Borges e } \\
\text { Zoboli }\end{array}$ & 2005 & BDENF/ Rev. Min. Enf & $\begin{array}{l}\text { Enfermagem em Saúde Coletiva: O } \\
\text { Diagrama de Controle Como } \\
\text { Estratégia de Ensino de Vigilância } \\
\text { Epidemiológica das Doenças } \\
\text { Transmissíveis }\end{array}$ \\
\hline
\end{tabular}

Quadro 4: Distribución de las bibliografías potenciales de categoría textual: "papel del profesional enfermero en el control de endemias" 
Gomes ${ }^{13}$ demostró en su artículo que las acciones del enfermero son las de participar en la coordinación de datos, en la producción de nuevas informaciones, proponer nuevas metodologías para su logro (estudios especiales e investigaciones epidemiológicas), realizar análisis de las limitaciones, seleccionar y aplicar las metodologías más adecuadas para el alcance de los objetivos propuestos por el programa que sea más adecuado al conocimiento de la enfermedad y su evolución; participar em la selección de alternativas, prioridades y colaborar en la elaboración, ejecutar los programas de control, así como evaluación del alcance de los objetivos propuestos. Concluye que, considerando el aspecto global del trabajo del enfermero y, en particular, sus acciones en la Vigilancia Epidemiológica y actuación en la administración de servicios, de asistencia de servicios o de asistencia de enfermería, la epidemiologáa tendrá un importante papel en estos procesos no solamente en los aspectos de prevención, de vigilancia, de enfermedades, o de ocurrencias, sino también en la evaluación y análisis de impactos de sus acciones.

Perdesolli, Antonialli e Vila ${ }^{14}$ objetivaron identificar y analizar los diferentes periodos en que el profesional enfermero fue incluido en la vigilancia epidemiológica a fin de demostrar la naturaleza de este trabajo y las transformaciones de las políticas de salud. Identificaron, entre otros, el papel del enfermero en el control de endemias, que trabaja con campañas, vacuna, inmunización, además de tener este profesional un predominio en los equipos de vigilancia epidemiológica de los distritos de salud.

Según el manual del Ministério da Saúde ${ }^{15}$, el papel del enfermero en el control de endemias es realizar el diagnóstico precoz, instituir el tratamiento adecuado e imediato y/o acompañarlo; desarrollar acciones educativas y de movilización social que puedan contribuir a las medidas de control individuales y colectivas, con impacto en la mejora de las situaciones identificadas. En las acciones educativas, el enfermero debe implementar medidas de prevención y, para ello, es preciso conocer las alteraciones ambientales, los lugares donde las personas viven, trabajan y duermen entre otros. El enfermero deberá también desarrollar medidas de protección individual, familiar y de la comunidad, desarrollar medidas de combate a vectores y otros microorganismos. En la prevención y promoción de la mejora de condiciones ambientales de la población, el enfermero, junto con su equipo que actúa en el control de endemias, deberá realizar acciones de educación en salud y de movilización social; movilizar a la comunidad para desarrollar medidas simples de manejo ambiental; rellenar y enviar al sector competente la ficha de notificación, conforme a la estrategia local; rellenar adecuadamente y enviar al sector competente el boletín de actividad diaria; participar en las reuniones de planificación y evaluar los resultados de las acciones de control. Es competencia de los enfermeros de los equipos de salud de familia y de la unidad básica de salud, identificar casos sospechosos, realizar diagnósticos precoces; realizar tratamiento inmediato y adecuado de los casos conforme al manual de cada endemia; orientar al paciente ante la necesidad de concluir el tratamiento; solicitar y orientar al paciente para retorno después de la finalización del régimen de tratamiento.

Nichiata, Borges e Zoboli ${ }^{16}$ destacan en su artículo que la actuación del enfermero junto a la vigilancia epidemiológica desarrolla acciones de investigación epidemiológica y diagnóstico situacional, de planificación e implementación de medidas de prevención, control y tratamiento. Destacan también que en el ámbito de la enfermería en salud comunitaria, la vigilancia epidemiológica viene siendo utilizada como una acción que necesita ser incorporada al cotidiano del trabajo del enfermero.

En este sentido, se demostró que la actuación del enfermero en el control de endemias se basa en la participación de la coordinación de los datos, en la producción de nuevas informaciones, en la realización de análisis de las limitaciones, en la selección de aplicación 
de las metodologías adecuadas para alcanzar los objetivos propuestos por el programa que sea más adecuado al conocimiento de la enfermedad y su evolución; en la participación de la selección de alternativas, prioridades y colaborar en la elaboración, en la ejecución de los programas de control, así como en la evaluación del alcance de los objetivos propuestos. El enfermero debe también tener una participación en los equipos de vigilancia epidemiológica de los distritos de salud, desarrollar acciones educativas y de movilización social que contribuyan a las medidas de control individuales y colectivas, con impacto en la mejora de las situaciones identificadas.

Para discutir esta categoría, Pedrazzani ${ }^{17}$ identificó las siguientes funciones como papel del enfermero en el control de endemias: educación en salud, asistencia de enfermería, administración de servicios y vigilancia epidemiológica.

Steagall-Gomes, Daisy Leslie $^{18}$, definen y delimitan las funciones del enfermero y del personal de enfermería. Sin embargo, determinar el límite de función de cada categoría en enfermería ha sido difícil en la práctica. Para estos autores las enfermeras de Centro de Salud estaban distribuídas en cinco funciones básicas, que eran ejecutadas en el siguiente orden de frecuencia: administrativas (68,5\%), asistencia de enfermería (67,5\%), enseñanza $(58,5 \%)$, asesoría $(40,0 \%)$ y por último investigación $(36,5 \%)$. Comparadas las frecuencias con que las enfermeras de Centro de Salud y las de la categoría jefe, con o sin cargo, ejercían la función asistencial, se verifica tener una inversión, por cuanto se preveía mayor frecuencia en relación a las primeras. Actualmente, surgen otras tendencias respecto a las funciones del enfermero, siendo la primera la que contempla la ampliación de sus atribuciones a fin de que desempeñe un mayor número de funciones médicas. La segunda postula que el enfermero debe profundizar en su propio campo, para convertirse en un especialista de enfermería.

\section{2 - La formación profesional del enfermero en el control de endemias}

En esta categoria, se incluyen tres artículos, un editorial y un manual que tratan sobre la formación del profesional enfermero en el control de endemias, o sea, la vivencia y la práctica de este profesional e el control de las endemias, conforme el cuadro 4.

\begin{tabular}{|c|c|c|c|}
\hline Autores (s) & Año & Base de datos/Revista & Título \\
\hline Gomes $^{13}$ & 1994 & $\begin{array}{l}\text { lacs/ Rev. Lat. Americana } \\
2 \mathrm{~N}^{\mathrm{o}} 2\end{array}$ & A Epidemiologia para o Enfermeiro \\
\hline $\begin{array}{l}\text { Perdesolli, } \\
\text { Antonialli e } \\
\text { Vila }^{14}\end{array}$ & 1998 & $\begin{array}{l}\text { BDENF/ Ver. } \quad \text { Lat. } \\
\text { Americana. V.6 } \mathrm{N}^{\circ} 5\end{array}$ & $\begin{array}{l}\text { O enfermeiro na Vigilância } \\
\text { Epidemiológica no município de } \\
\text { Ribeirão Preto }\end{array}$ \\
\hline $\begin{array}{l}\text { Ministério } \\
\text { da saúde }\end{array}$ & 2002 & $\begin{array}{l}\text { Ministério da Saúde/ } \\
\text { Fundação Nacional de Saúde }\end{array}$ & $\begin{array}{l}\text { Ações de Controle de Endemias/ } \\
\text { Manual de Agente Comunitário de } \\
\text { Saúde e Agente de Controle de } \\
\text { Endemias }\end{array}$ \\
\hline $\begin{array}{l}\text { Nichiata, } \\
\text { Borges } \\
\text { Zoboli }^{16}\end{array}$ & 2005 & $\begin{array}{l}\text { BDENF/ Rev. Min. Enf V.9 } \\
\text { N } 4^{\circ}\end{array}$ & $\begin{array}{l}\text { Enfermagem em Saúde Coletiva: o } \\
\text { Diagrama de Controle Como } \\
\text { Estratégia de Ensino de Vigilância } \\
\text { Epidemiológica das Doenças } \\
\text { Transmissíveis }\end{array}$ \\
\hline Oliveira $^{19}$ & 2008 & $\begin{array}{l}\text { BDENF/ Epidemiologia } \\
\text { Serviços e Saúde (editorial) } \\
\text { V. } 17 n^{\circ} 2\end{array}$ & $\begin{array}{l}\text { Desafios para o Controle e Diagnose } \\
\text { de Doenças Endêmicas e o Papel da } \\
\text { Pesquisa }\end{array}$ \\
\hline
\end{tabular}

Quadro 5: Distribución de las bibliografías potenciales de la categoría textual: "la formación del profesional enfermero en el control de endemias." 
Gomes $^{13}$ en su artículo, relata que la enseñanza de epidemiología desarrolla habilidades para establecer prioridades dentro de los límites de los recursos existentes, lo que capacita al enfermero para sus acciones en las actividades en la vigilancia epidemiológica (VE). La epidemiología se configura así en un instrumento valioso para la utilización de los enfermeros en su práctica, ya sea en la enseñanza, en los servicios, en sus actividades administrativas específicas y/o en las investigaciones. Su contenido deberá ser ofertado en la formación profesional del enfermero por considerarse un conocimiento necesario para su práctica profesional, haciendo las acciones de enfermería más efectivas y conscientes.

Perdesolli, Antonialli e Vila ${ }^{14}$ identificaron que las actividades de vigilancia epidemiológica desarrolladas por el profesional de enfermería requieren conocimientos específicos de epidemiología, control de enfermedades y problemas de salud, gerencia de programas (planificación, evaluación y coordinación), orientación técnica de las acciones de vigilancia epidemiológica y vacunación, necesitando el enfermero tener formación específica en Salud Comunitaria. El predominio del enfermero en la Vigilancia Epidemiológica necesita de un profesional con conocimiento más especializado, formación más exhaustiva, desarrollo de habilidades técnicas de enfermería, mayor capacitación y conocimiento de medidas de control (prevención), a fin de evitar la aparición de enfermedades.

Según el Ministerio de Salud ${ }^{15}$, en su manual, la educación de los agentes ha de ser continuada, con diversas técnicas, que capacite para unir lo creativo con lo disponible en sus comunidades. La capacitación orienta a los agentes en cómo llegar a las casas de las personas, hablar con ellas, entender el momento de cada una, escuchar, y, sobre todo, desarrollar la percepción de lo que está ocurriendo en aquellas casas, con aquellas personas.

Nichiata, Borges e Zoboli ${ }^{16}$ resaltan en su artículo, que esta estrategia de enseñanza en la formación del alumno de graduación en enfermería complementa y sedimenta los conceptos básicos utilizados em la práctica de vigilancia epidemiológica y vigilancia en la salud, siendo evaluada positivamente por los alumnos.

Oliveira $^{19}$, en su editorial, destaca que la evaluación de intervenciones de control de enfermedades y su efectividad dependen también de la calidad de los métodos adoptados, llama la atención de los profesionales de salud y de los gestores del sistema para la necesaria participación de la salud, juntamente con las instituciones de pesquisas, iniciativas de desarrollo científico y tecnológico. Además de hacer que los servicios de salud alcancen los mejores resultados (encontrados).

En este aspecto, la formación profesional es muy importante para que el enfermero actúe en el control de endemias de forma efectiva y eficaz. Se destaca que la enseñanza de epidemiología desarrolla habilidades para establecer prioridades dentro de los límites de los recursos existentes, capacitando al enfermero para sus acciones en las actividades de vigilancia epidemiológica (VE).

Por tanto, es importante que el enfermero tenga una formación específica en Salud Comunitaria.

Pedrazzani ${ }^{17}$ en su atrículo establece que la categoría formación del enfermero fue verificada en los docentes responsables de la enseñanza de endemias en el 66,60 por ciento de los Cursos de Graduación en Enfermería del Estado de São Paulo. Las informaciones sobre el desarrollo de su práctica en el área de endemia se obtuvieron a través de los 
propios enfermeros en las unidades ambulatorias de la red de servicios públicos del Estado; se utilizó un formulario propio para el levantamiento de datos, que tuvo lugar durante el primer semestre de 1989. Los resultados obtenidos indican un deficiencia en la enseñanza de endemia, siendo necesario dar mayor importancia al tema, a través de una reformulación del mismo en los currículos de los Cursos de Graduación en Enfermería.

Steagall-Gomes, Daisy Leslie ${ }^{18}$, en su artículo, describen la apreciación de las enfermeras sobre su formación acadêmica, mostrando que para $73,5 \%$ la enseñanza teórica fue suficiente y para $67,5 \%$ la práctica también lo fue. Las que respondieron ser insuficiente atribuyeron el hecho a la insuficiencia de cursos, a lo inadecuado de la enseñanza y del currículo sobre refiriéndose a la poca importancia prestada a la Salud Comunitaria; sobre la práctica se criticó las etapas. Como $28,5 \%$ del grupo no estaba habilitado en enfermería de salud comunitaria, se justifican las respuestas considerando los cursos como insuficientes. Se constató también, que había una limitación de disponibilidad de material bibliográfico, escasez de oportunidad para cursos y encuentros de interés de la categoría, dificultad monetaria, falta de interés del propio servicio en ofrecer medios para actualizarse al profesional, entre otros.

\section{4- CONSIDERACIONES FINALES}

Al traer el asunto para discusión, se percibió una deficiencia en las publicaciones científicas, evidenciando la necesidad de más investigaciones con enfásis en la valoración del profesional de enfermería como instrumento articulador del proceso, siendo necesario dar mayor importancia al tema, a través de una reformulación en los currículos de los Cursos de Graduación en Enfermería, introduciendo estrategias de enseñanza que valoren y muestren la verdadera importancia de la epidemiología en el papel del enfermero para el control de endemias.

Se concluye que el enfermero incluído en esta área deberá proveerse de técnicas de prevención y promoción de la salud, atendiendo a una mejor calidad de vida para la comunidad. Ello tendrá como propuesta de acciones educativas el entrenamiento de sus agentes, tendiendo siempre a analizar cómo la enfermedad se distribuye según las características de las personas, lo que determina su ocurrencia, elaborar las medidas a ser tomadas a fin de prevenir y controlar el saber hasta qué punto las medidas contribuirán a la prevención de la enfermedad.

\section{REFERENCIAS BIBLIOGRÁFICAS}

1- Medronho RA. Epidemiologia. São Paulo, Atheneu, 2007.

2- Rouquayrol ZM.; Almeida F. Introdução a Epidemiologia. 4ํe edição revisada e ampliada: Guanabara koogan, 2006.

3- Silva Luiz Jacintho da. O controle das endemias no Brasil e sua história. Cienc. Cult. [Serial on the Internet]. 2003 Jan [cited 2008 Oct 24]; 55(1): 44-47. Available from: http://cienciaecultura.bvs.br/scielo.php?script=sci_arttext\&pid=S0009-

$67252003000100026 \&$ Ing=en.

4- Benchimol JL. Dos micróbios aos mosquitos. Febre amarela e a revolução pasteuriana no Brasil/ Editora: Fio Cruz/UFRJ, 1999.

5- Rodrigues C. Acidade e a morte: a febre amarela e seu impacto sobre os costumes fúnebres. Revista História, Ciências, Saúde (Manguinhos); 6:53- 80. 1999

6- Almeida M.; Dantes MAM. O serviço sanitário de São Paulo, a saúde pública. Revista Espaços da ciência do Brasil. 1800-1930. Rio de Janeiro, 2001. 
7- Figueredo NMA (Org). Método e metodologia na pesquisa científica. São Paulo: Yendis, 2007.

8- Oliveira SL. Tratado de metodologia científica. São Paulo, Editora pioneira, 1999.

9- Polit DF; Beck CT; Humgler BP. Fundamentos de pesquisa em enfermagem: Métodos, avaliação e utilização. Porto Alegre: Artmed, 2004.

10- Cervo AL; Bervian PA. Metodologia científica. São Paulo: Prentice Hall, 2002.

11- Minayo MCS, ( Org.). Pesquisa social: teoria, método e criatividade. Rio de Janeiro: Vozes, 1994.

12- Gil AC.; Como elaborar projetos de pesquisa. 4ํe edição. São Paulo: Atlas, 2006

13- Gomes. A epidemiologia para o enfermeiro. Revista Latino Americana volume 2, n:2, 1994

14 - Perdesolli; Antonialli, Vila. O enfermeiro na vigilância epidemiológica no município de Ribeirão Preto. Revista Latina Americana volume6, n: 51998.

15- Brasil MS. Ações de controle de endemias/Manuel de agente comunitário de saúde e agente de controle de endemias. Manual do Ministério da Saúde, 2002.

16- Nichiata; Borges; Zoboli. Enfermagem em saúde coletiva: o diagrama de controle como estratégia de ensino de vigilância epidemiológica das doenças transmissíveis. Revista Mineira de Enfermagem volume 9, n:4, 2005

17- Pedrazzani; Silva E. O ensino e a prática do enfermeiro em Hanseníase. Tese apresentada a Universidade de São Paulo, pág: 161, 1990.

18- Steagall C.; Leslie D. Identificação do enfermeiro de saúde pública na força de trabalho de enfermagem de saúde pública no departamento Regional de saúde de Ribeirão Preto. Revista Saúde Pública volume 24, №3, p: 224-231, 1990.

19- Oliveira. Desafios para o controle e diagnose de doenças endêmicas e o papel da pesquisa. Editorial Epidemiologia e Saúde volume: 17 nํ2, 2008.

ISSN 1695-6141

@ COPYRIGHT Servicio de Publicaciones - Universidad de Murcia 